\title{
Structure of hexapod 3D packings: understanding the global stability from the local organization
}

\author{
Jonathan Barés ${ }^{1,2, \star}$, Yuchen Zhao ${ }^{2}$, Mathieu Renouf ${ }^{1}$, Karola Dierichs ${ }^{3}$, and Robert Behringer ${ }^{2}$ \\ ${ }^{1}$ Laboratoire de Mécanique et Génie Civil, Université de Montpellier, CNRS, Montpellier, France \\ ${ }^{2}$ Department of Physics \& Center for Nonlinear and Complex Systems, Duke University, Durham, North Carolina 27708, USA \\ ${ }^{3}$ Institute for Computational Design, University of Stuttgart, Stuttgart, Germany
}

\begin{abstract}
.
Aggregates of non-convex particles have shown to be particularly stable which makes them good candidates to design new lightweight and reversible structures. However, few is known about the fundamental reason of their stability. In this paper we presents a novel experimental method to investigate the local structure of piles made of hexapod particles. This method is based on X-ray scanning and on an accurate homemade particle detection code. It permits to get the position and orientation of each particle as well as to detect their contact points. Measurement of the coordination numbers, statistics of the contact positions and local density evaluation for different packing configurations show a good agreement with the previous studies carried out at the global scale and permits to explain the main local mechanisms leading to stable structures.
\end{abstract}

\section{Introduction}

The use of aggregates made from non-convex particles or particles with anisotropic contact laws is an emerging area for both the research in physics [1-7] and the design of functionalized materials by tuning local properties of the grains [8-11]. This new field in granular matter science has already shown to be extremely promising for future lightweight and reversible architecture [12]. It permits to design easy-to-shape and remove strong structures. Indeed the shape of the grains [2, 13, 14] and the contact anisotropy [8] have been recently used to help crossing the jamming transition and to increase the stability/rigidity of granular systems. Different particle shapes, such as long and thin rods [15], staples [4], Z-shaped [6] and starshaped $[2,5]$ particles have been shown to bring additional rigidity to $3 \mathrm{D}$ packings, compared with spherical shapes [16].

Two of the most striking characteristics of non-convex designed granulates, in terms of the granular system and possible design applications are their ability to form vertical structures with a $90^{\circ}$ angle of repose, and to sustain small tilting or loading perturbations [2]. Even if this tremendous stability illustrated in the fig.1-A has already been evidenced in several experiments $[2,5,17,18]$ and if packing of such particles has been widely studied [1921], few is know about the fundamental reasons of why non convex particle piles are much more stable than bead columns [16, 22, 23].

In order to understand the origin of this rigidity, in this paper, we investigate the local organization of columns

\footnotetext{
^e-mail: jb@jonathan-bares.eu
}

made of star-shape or hexapod particles (see fig.1-A) using $\mathrm{X}$-ray CT-scan. We have performed experiments by randomly pouring identical star-shape particles $(2.5$ to $10 \mathrm{~cm}$ wide) into hollow cylinders, and removing the cylinders to get stable star columns, for different preparation conditions. Then, by mean of a X-ray CT-scan and a home made post-processing code, we have reconstructed the full structure of the packing: (i) position, (ii) orientation and (iii) contact points of each particles with a very good accuracy $(0.1 \mathrm{~mm})$. We have analysed the coordination number and the distributions of the contact point positions.

In a first part we present the experimental set-up to prepare and scan hexapod piles. In a second part the method to measure the position, orientation and contact points for each particle is explained. Then, analysis tools are detailed before presenting the results.

\section{Experimental method}

The cylindrical columns have been prepared in two different ways and we used three kinds of hexapods particles as shown in fig.1-A. These last consisted of six orthogonal beams with square cross section, that tapered from a thickness of $2 \mathrm{~mm}$ at the center of the particle to $1 \mathrm{~mm}$ at the tips. The end-to-end size, $L$, varied between $2.5 \mathrm{~cm}$, $5 \mathrm{~cm}$ and $10 \mathrm{~cm}$. They are made of laser-sintered white nylon PA2200, which has a friction coefficient $1.0 \pm 0.3$. In this paper we will only talk about $2.5 \mathrm{~cm}, 5 \mathrm{~cm}$ star packings because for $10 \mathrm{~cm}$ stars the number of particles in the structure is not large enough to give statistically meaningful results. 
A
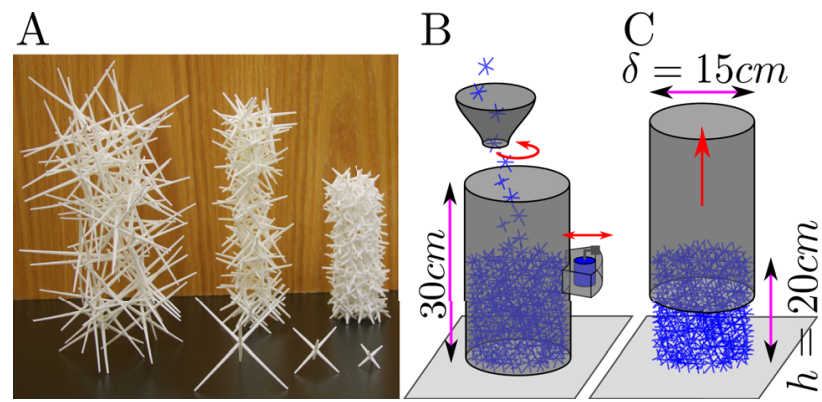

Figure 1. A: Columns of $2.5 \mathrm{~cm}, 5 \mathrm{~cm}$ and $10 \mathrm{~cm}$ wide hexapods made of laser sintered nylon PA2200. Hexapods can form very stable structures capable of sustaining their own weight, tilt and external loading [2]. B: Cartoon where the non-convex particles are dropped one-by-one from an overhead hooper in a cylinder of diameter $15 \mathrm{~cm}$. The system can be vibrated by an eccentric, driven by a DC motor which is attached to the cylinder. C: The cylinder is then carefully and slowly removed by sliding lifting it vertically. Hexapods form a stable pile of height $\sim 20 \mathrm{~cm}$.

The protocol to make particle piles consisted in filling thin-walled PVC tube with particles by dropping them one-by-one at a steady rate from an overhead hopper as shown in fig.1-B. The inner diameter of the PVC tube was $15 \mathrm{~cm}$. In some cases, during the filling process, the system could be vibrated by an eccentric that was attached to the outside of the tube as presented in the fig.1-B. This has been shown to increase the pile stability [2]. We tuned the speed of the motor to create $\mathrm{a} \sim 1700 \mathrm{~m} / \mathrm{s}^{2}$ cyclic acceleration of the $16 \mathrm{~g}$ eccentric. As shown in fig.1-C, the tube was then carefully and slowly removed by lifting it vertically. Friction between the particles and the cylinder was low enough not to significantly perturb particles inside the cylinder. This protocol is similar to what has been used by [2].

The hexapod column was then set inside a X-ray microCT scanner Nikon XT-H225 and scan with a X-ray source of $185 \mathrm{kV}$ and $235 \mu \mathrm{A}$. Raw data were then postprocessed using Nikon's Feldkamp [24] cone based CT algorithm to get 2000 density slices of size $2000 \times 2000$, with a spacial resolution of $0.1 \mathrm{~mm}$ and a 16bits digital resolution. A slice in the middle of the column is shown in fig.2-A.

\section{Data post-processing}

Collecting the density slices we then formed a full 3D density matrix $M_{0}$ (see cartoon for two particles in fig.2-B) which we post-processed with a homemade Python algorithm to extract the position $x, y, z$ and the orientation $\theta_{1}$, $\theta_{2}, \theta_{3}$ of each particle. Because of the large dimension of the density matrix we first resize it to $M$ a $400 \times 400 \times 400$ binarized matrix choosing a $60 \%$ density threshold $(100 \%$ is the highest density, $0 \%$ the lowest). A virtual smaller binary matrix $m\left(\theta_{1}, \theta_{2}, \theta_{3}\right)$ is then formed. As presented in fig.2-C this matrix is null everywhere except for the pattern of an hexapod centered in the middle of the matrix and oriented along three axis: $\theta_{1}, \theta_{2}, \theta_{3}$.
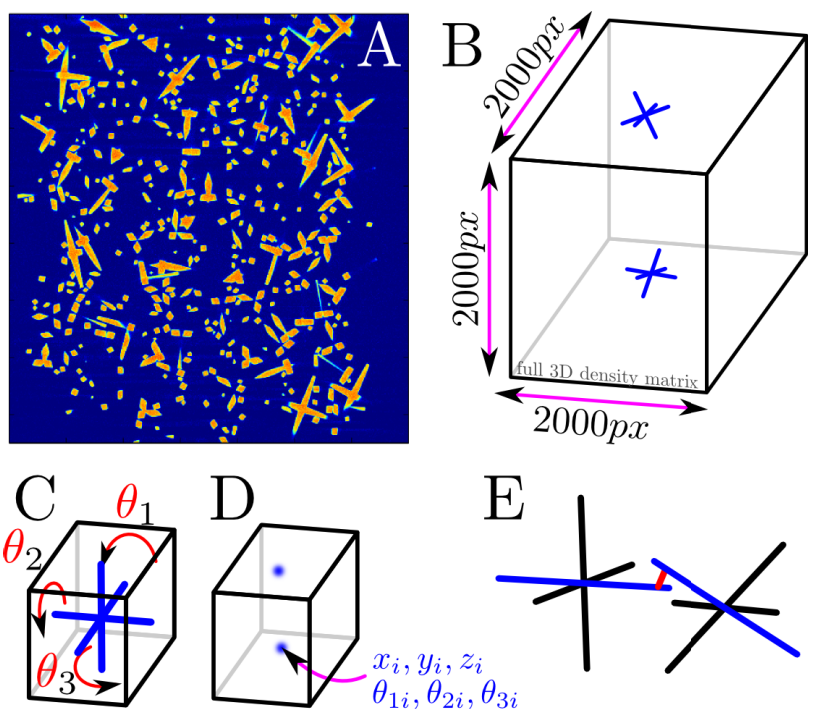

Figure 2. A: Slice of a density matrix in the middle of the hexapod pile. Blue is for low density (surrounding air) while red is for high density (particle nylon). B: Cartoon of a density matrix with only 2 hexapods. Matrices are 16 bits $2000 \times 2000 \times 2000$ voxels. C: Cartoon of the convolution density matrix. This matrix is used for hexapod detection by convolving it with the density matrix (B) for particle with a certain orientation: $\theta_{1}, \theta_{2}, \theta_{3}$. D: Synthesized convolved matrix. Blue high value islands show position and give orientation of the particles in the density matrix (B). See text for details. E: Cartoon of two neighbour particles. The red segment show the shortest distance between the two blue hexapod arms.

Taking advantages of the particle symmetry, for all the possible families of direction with $\theta_{i}$ going from $0^{\circ}$ to $90^{\circ}$ with $2^{\circ}$ steps (91125 families), we convoluted the binarized density matrix $M$ with $m\left(\theta_{1}, \theta_{2}, \theta_{3}\right)$ to get a convolution matrix $C\left(\theta_{1}, \theta_{2}, \theta_{3}\right)$ of the same size as $M$. This computation took $80 \mathrm{~h}$ parallelized on a 60 nodes cluster. Then, a maxima matrix $D$ of the same size as $M$ is formed. For each coordinate $i, j, k$ it took the maximum convolution value $C_{i, j, k}\left(\theta_{1}, \theta_{2}, \theta_{3}\right)$ over the 91125 possible orientations and keep the record of the corresponding $\left(\theta_{1}, \theta_{2}, \theta_{3}\right)$ family. As presented in the cartoon fig.2-D, this matrix shows high convolution values $3 \mathrm{D}$ islands at the position of the hexapod center. Using this property, a 3D local maxima research algorithm applied on the matrix $D$ gave the position of each hexapod $x_{i}, y_{i}, z_{i}$ and the record of their orientation gave $\theta_{1 i}, \theta_{2 i}, \theta_{3 i}$.

However, this measurement is not accurate enough since it has been done on a density matrix whose size has been divided by $5(0.5 \mathrm{~mm}$ accuracy on the particle center position) and orientation is only $2^{\circ}$ accurate. To improve the measurement, for each detected particle center a submatrix of the full density matrix $M_{0}$ is extracted and binarized. A larger virtual binary matrix is made and a Powell optimization algorithm was implemented to improve the match between both matrices and give more accurate position $\left(0.1 \mathrm{~mm}\right.$ accuracy) and orientation $\left(0.5^{\circ}\right.$ accuracy $)$ values. This part of the algorithm is reminiscent of the method used by [25]. 

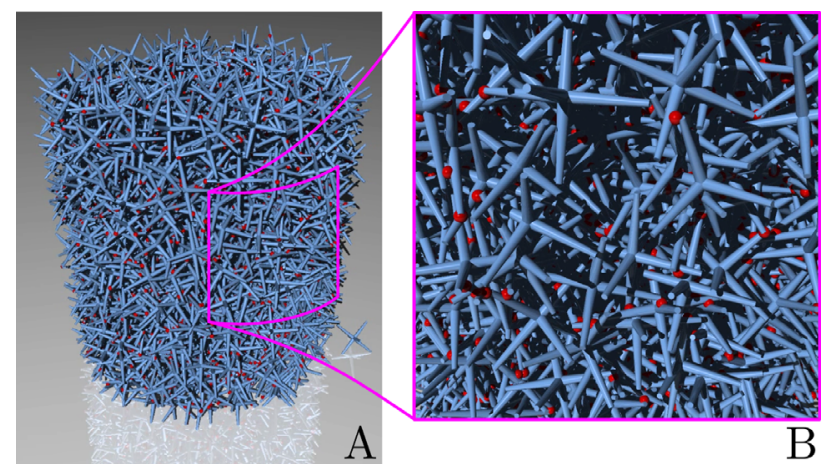

Figure 3. A: Full reconstruction of an hexapod packing from the X-ray scan of a non vibrated column of $2.5 \mathrm{~cm}$ stars. The drawing has been generated using Vapory, the Pov-Ray wrapper in Python, knowing the hexapod geometry, and the measured position and orientation. Red spheres show the position of the contacts. B: Zoom in the specified area presented of A. A complete movie of the reconstruction for each particle size is given here: https://www.youtube.com/watch?v=2HUUAPet2rs.

Contact between the hexapods arms were finally detected from the particle position and orientation, and the density matrix $M$. As presented in fig.2-E, for each arm of each particle, the distance between the segment formed by the arm and the the segment formed by each arm of the neighbour particles was computed. If this distance were lower than $4.5 \mathrm{~mm}$, a $10 \times 10 \times 10$ submatrix (corresponding to $1 \mathrm{~mm}^{3}$ ) centred around the hypothetical point of contact was extracted from the density matrix $M$. This submatrix was binarized as previously and the number of connected domains computed. If 2 distinct domains were observed there was no contact. If there was only one large domain the two arms were in contact. This method gives contacts with a $0.1 \mathrm{~mm}$ threshold accuracy. Further investigations are currently carried out to shows that this threshold is small enough not to detect too much fake contacts. A reconstruction of the 3D packing as well as the position of the contact is presented in fig.3.

\section{Results}

A coordination number (average number of contact per grain) of 6.58 have been measured for non vibrated $2.5 \mathrm{~cm}$ particles. This value is larger than the theoretical coordination 6 at the limit of stability, so this explain the stability of such a system. When particles are vibrated, as emphasized by [2], we expect to have a more stable structure. Indeed, we measure a coordination number of 7.13 for such a configuration. For particle with longer arms $(5 \mathrm{~cm})$ we expect something even more stable [2] which corresponds with the coordination number we measured: 9.18. Hence, this explain the increase is stability for vibrated system and large particle observed by [2] but also quantify this stability by giving the hyperstaticity of the different configurations.

In fig.4-A, we present the probability density function (PDF) of the contact position along a particle arm. For each system configuration, $2.5 \mathrm{~cm}, 5 \mathrm{~cm}$ particles, vibrated
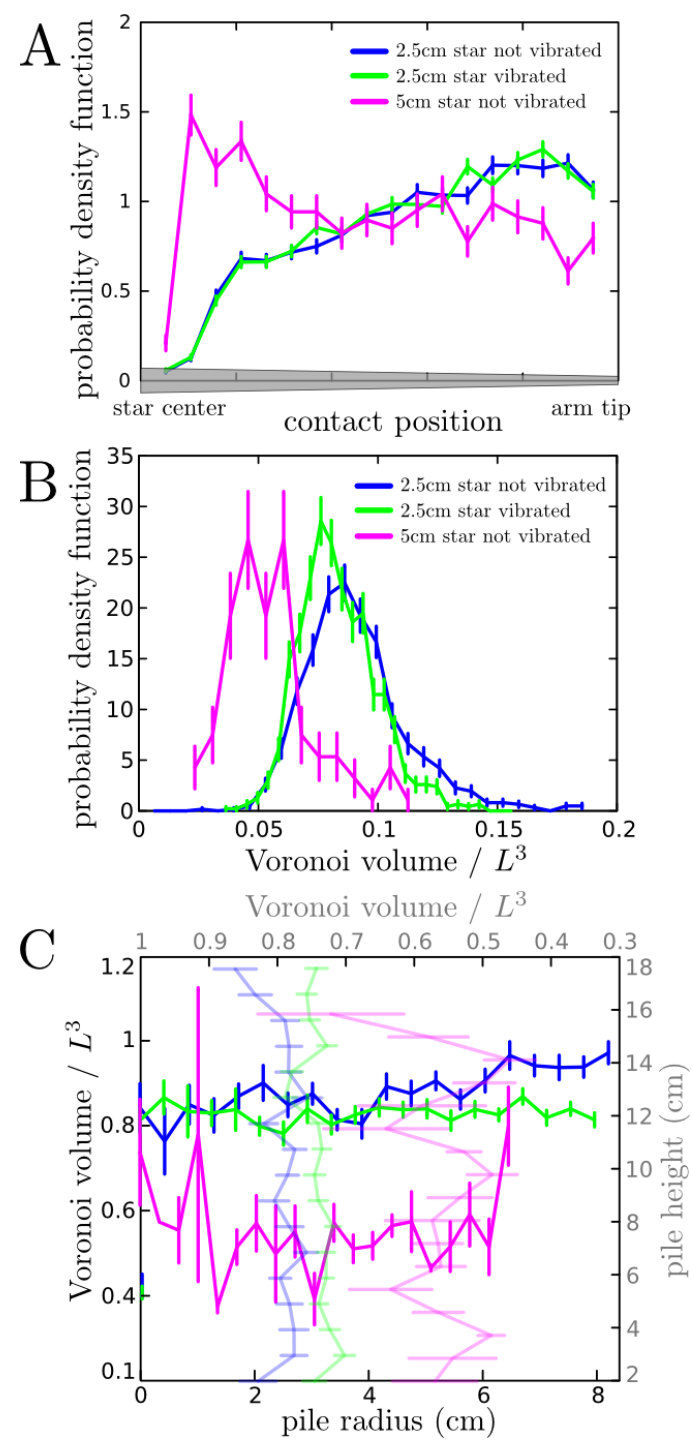

Figure 4. A: Probability density function of the position of a contact along a particle arm for the different system configurations: $2.5 \mathrm{~cm}, 5 \mathrm{~cm}$ particles, vibrated and non-vibrated. $x$-axis is rescaled to compare $2.5 \mathrm{~cm}$ and $5 \mathrm{~cm}$ stars. Vibration has no effect on the average contact position but the longer the arms, the closer the contact points are from the particle center. B: Probability density function of the particle local density. For each configuration the probability density function of the rescaled Voronoi volume around each star is plotted. The larger the particles or the more vibrated the system, the wider the statistical repartition. C: Averaged scaled Voronoi volume along the radial (horizontal plot) and height (vertical plot) directions for the 3 different packings. The particle density just slightly increases radially. Vertical error-bars give the $95 \%$ confidence intervals of the measurements.

and non-vibrated, for each arm of each particle, the distance of the contacts to the particle center has been measured. Then the PDF of this distance rescaled with the arm length $L$ has been computed. We see that in the case of large particles, contacts are homogeneously spread along the whole arm, with an over-representation close to the particle center. On the contrary, for shorter particles the contacts are more concentrated at the arm tip. This explain the more important stability of large particle piles since 
hexapods are more intricated so more difficult to destabilize.

Fig.4-B present the PDF of the local packing fractions. For each system configuration, from the position coordinate of the particles, we have computed the 3D Voronoi tesselation. The volume of each tile is measured and rescaled with the an effective hexapod volume $L^{3}$. The PDF of this quantity is then plotted. For each case it vanishes before $10 \%$, then presents a sharp peak and vanishes more progressively for larger volumes. We remarks that the vibration does not seems to have any significant effect on the statistical repartition of the local particle densities.However, the PDF peaks correspond to a larger scaled volume for $2.5 \mathrm{~cm}$ particles than for $5 \mathrm{~cm}$ ones. Indeed, for larger hexapods, the harm aspect ratio is thinner so particle can entangle deeper. It is remarkable to notice that this property corresponds with a more stable system configuration.

Fig.4-A shows the evolution of the average local density measured as the volume of the Voronoi tiles scaled with an effective hexapod volume $L^{3}$. This quantity has been averaged vertically and circularly to give the radial evolution of the local particle density for each packing configuration. We see that the pile is slightly denser in the inside and for larger particles but constant for $5 \mathrm{~cm}$ ones. Averaging along the vertical dimension, we get the vertical evolution of the density. Surprisingly, within the error-bar, there is no variation of the density as a function of the pile height.

\section{Conclusions}

We presented an experimental method to investigate the local structure of aggregates made from non-convex particles. This method is based on X-ray scanning and on an efficient and $0.1 \mathrm{~mm}$ accurate homemade particle detection code using parallelized matrix convolutions. This permits to measure the position and orientation of different sized hexapods forming large piles in vibrated and nonvibrated configurations. This local scale information is in good agreement with the macroscopic stability measurement made by [2]. Moreover, contact position statistics and local packing fraction variability provided interesting information about the effect of the pile preparation. However, these experimental results do not permit to detect the exact nature of the contact (point, line or plane) nor to measure the force. Hence, to test the accuracy of our experimental measurement as well as to improve the contact analysis, numerical simulations will be carried out.

\section{Acknowledgements}

This work was supported by the Labex NumEv (anr10-labx-20), the William M. Keck Foundation, by NSF grant DMR1206351 and NASA grant NNX15AD38G and ITASCA Educational Partnership Program.

\section{References}

[1] S. Keller, H.M. Jaeger, Granular Matter 18, 29 (2016)

[2] Y. Zhao, K. Liu, M. Zheng, J. Barés, K. Dierichs, A. Menges, R.P. Behringer, Granular Matter 18, 24 (2016)

[3] M.Z. Miskin, H.M. Jaeger, Soft Matter 10, 3708 (2014)

[4] S. V. Franklin, Europhysics Letters 106, 58004 (2014)

[5] K. Dierichs, A. Menges, Granular Matter 18, 1 (2016)

[6] K.A. Murphy, N. Reiser, D. Choksy, C.E. Singer, H.M. Jaeger, Granular Matter 18, 1 (2016)

[7] P. Aejmelaeus-Lindström, J. Willmann, S. Tibbits, F. Gramazio, M. Kohler, Granular Matter 18, 1 (2016)

[8] M. Cox, D. Wang, J. Barés, R.P. Berhinger, Europhysics Letters (2016)

[9] M. Z. Miskin, H. M. Jaeger, Nature Materials 12, 326 (2013)

[10] B. Saint-Cyr, J.-Y. Delenne, C. Voivret, F. Radjai, P. Sornay, Physical Review E 84, 041302 (2011)

[11] E. Brown, A. Nasto, A. G. Athanassiadis, H. M. Jaeger, Physical Review Letters 108, 108302 (2012)

[12] K. Dierichs, A. Menges, Architectural Design 85, 86 (2015)

[13] A. G. Athanassiadis, M. Z. Miskin, P. Kaplan, N. Rodenberg, S. H. Lee, J. Merritt, E. Brown, J. Amend, H. Lipson, H. M. Jaeger, Soft Matter 10, 48 (2014)

[14] K. C. Smith, M. Alam, T. S. Fisher, Physical Review E 82, 051304 (2010)

[15] M. Trepanier, S. Franklin, Physical Review E 82, 011308 (2010)

[16] E. Lajeunesse, A. Mangeney-Castelnau, J.P. Vilotte, Physics of Fluids 16, 2371 (2004)

[17] N. Gravish, S. V. Franklin, D. L. Hu, D. I. Goldman, Physical Review Letters 108, 208001 (2012)

[18] S. V. Franklin, Physics Today 65, 70 (2012)

[19] K. Miszta, J. de Graaf, G. Bertoni, D. Dorfs, R. Brescia, S. Marras, L. Ceseracciu, R. Cingolani, R. van Roij, M. Dijkstra, Nature Materials 10, 872 (2011)

[20] J. de Graaf, R. van Roij, M. Dijkstra, Physical Review Letters 107, 155501 (2011)

[21] Y. Jiao, F. H. Stillinger, S. Torquato, Physical Review E 79, 041309 (2009)

[22] G. Lube, H. Huppert, R. Sparks, A. Freundt, Physical Review E 72, 041301 (2005)

[23] G. Lube, H.E. Huppert, R. S. J. Sparks, M. A. Hallworth, Journal of Fluid Mechanics 508, 175 (2004)

[24] L.A. Feldkamp, L.C. Davis, J.W. Kress, Journal of the Optical Society of America A 1, 612 (1984)

[25] M. Neudecker, S. Ulrich, S. Herminghaus, M. Schröter, Physical review letters 111, 028001 (2013) 